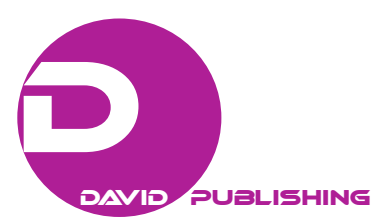

\title{
Spectral Expansions of Cosmological Fields
}

\author{
Anatoliy Malyarenko \\ Mälardalen University
}

\begin{abstract}
We give a review of the theory of random fields defined on the observable part of the Universe that satisfy the cosmological principle, i.e., invariant with respect to the 6-dimensional group $\mathcal{G}$ of the isometries of the timeslice of the Friedmann-Lemaitre-Robertson-Walker standard chart. Our new results include proof of spectral expansions of scalar and spin weighted $\mathcal{G}$-invariant cosmological fields in open, flat, and closed cosmological models.
\end{abstract}

Keywords: Random field, spectral expansion, spin weighted harmonic.

\section{Introduction}

The space mission Euclid, planned for launch in 2019, is expected, just to mention a few, to measure the shapes of 1.5 billion galaxies. In mathematical terms, Euclid will observe a single realisation of a random section of a spin bundle over a ball of radius about 10 billion light years. Analysis of Euclid data should base on spectral expansions of different cosmological fields. In this paper, we make attempt to review the known expansions and to present some new results.

In Section 2, we review the essential axioms, definitions, and results of the Standard Cosmological Model. We consider open, flat, and closed Friedmann-Lemaitre-Robertson-Walker cosmological models. The time slice $X$ of each of the above models has 6-dimensional group of isometries $\mathcal{G}$, where $\mathcal{G}=S O_{0}(1,3)$ for the open model, $\mathcal{G}=I S O(3)$ for the flat model, and $G=S O(4)$ for the closed model.

Section 3 introduces scalar cosmological fields, both deterministic and stochastic. In particular, Subsection 3.1 reviews expansions of deterministic cosmological fields in eigenfunction of the $\mathcal{G}$-invariant Laplace-Beltrami operator. The radial coefficients $R_{k \ell}(\chi)$ and $R_{\omega \ell}(\chi)$ of the above expansions are used in Subsection 3.2 to deduce spectral expansions of $\mathcal{G}$-invariant stochastic fields. The above expansions are proved in Theorem 1 similarly for all three models, using tools from harmonic analysis.

In Section 4 we first motivate introduction of spin stochastic cosmological fields, using the theory of the effective lensing potential. After short review of spin weighted spherical harmonics, we prove Theorem 2, that gives the spectral expansion of the convergence, shear, and of the two gravitational flexions cosmological fields.

I am grateful to Professor Domenico Marinucci for useful discussions on cosmology.

\section{Preliminaries from Cosmology}

According to modern cosmology, the Universe is governed by Einstein's equations. Solution of the above

Corresponding author: Anatoliy Malyarenko, Professor, Division of Applied Mathematics, School of Education, Culture and Communication. E-mail: anatoliy.malyarenko@mdh.se. 
equations is beyond the capabilities of modern computers. Cosmologists use another approach. They start from a simple exactly solvable model as a background solution and then add perturbations order by order.

The background solution is described as follows. The Universe is a 4-dimensional Lorentzian manifold $M$ satisfying the following two axioms.

1. The cosmological principle: at each epoch, the Universe presents the same aspect from every point.

2. The Weyl's postulate after Weyl (1923):

"The particles of the substratum (representing the nebulae) lie, in spacetime of the cosmos, on a bundle of geodesics diverging from a point in the (finite or infinite) past."

The "substratum" is the underlying fluid defining the overall kinematics of a system of galaxies. The galaxies follow their geodesics. Only one geodesic is passing through each point in $M$, except at the origin. This allows one to define the concept of fundamental observer, one for each geodesic. Each of these is carrying a standard clock, for which they can synchronise and fix a cosmic time.

For each fundamental observer, there exists a standard chart $(t, \chi, \theta, \varphi)$ of the manifold $M$ centred at the observer in which the metric has the Friedmann-Lemaittre-Robertson-Walker form:

$$
d s^{2}=-c^{2} d t^{2}+a^{2}(t)\left[d \chi^{2}+f_{K}^{2}(\chi)\left(d \theta^{2}+\sin ^{2} \theta d \varphi^{2}\right)\right]
$$

where the function $f_{K}$ depends on the curvature $K$ as

$$
f_{K}(\chi)= \begin{cases}(-K)^{-1 / 2} \sinh (\sqrt{-K} \chi), & K<0, \\ \chi, & K=0, \\ K^{1 / 2} \sin (\sqrt{K} \chi), & K>0,\end{cases}
$$

and where $t$ is the cosmic time, $\chi$ the comoving distance, $\theta$ and $\varphi$ are spherical coordinates on the celestial sphere, $c$ is the speed of light in vacuum, $a(t)$ is the scale factor. The comoving distance from the observer to a galaxy is the distance the galaxy will have from the observer when it is as old as the observer now. The physical distance is the product of the scale factor and the comoving distance. We choose the scale factor to be dimensionless and set $a\left(t_{0}\right)=1$, where $t_{0}$ is the value of the cosmic time now. Thus, the comoving distance $\chi$ has the dimension of length, while $K$ has the dimension of inverse length squared. The velocity of a galaxy with respect to a fundamental observer is called the peculiar velocity.

The time slice $D_{t}$ of the domain $D$ of the above chart is the Riemannian manifold $X$ with metric $a^{2}(t)\left[d \chi^{2}+f_{K}^{2}(\chi)\left(d \theta^{2}+\sin ^{2} \theta d \varphi^{2}\right)\right]$. The geodesic lines of fundamental observers are orthogonal to the time slices. The cosmological principle implies that each time slice $X$ is the 3-dimensional space of constant curvature. When the curvature is negative, the model is called open, the group of isometries of $X$ is $\mathcal{G}=S O_{0}(1,3)$, and $X=\mathbb{H}^{3}$, the hyperbolic space. When the curvature is equal to 0 , the model is called flat, the group of isometries of $X$ is $\mathcal{G}=I S O(3)$, and $X=\mathbb{R}^{3}$. Finally, when the curvature is positive, the model is called closed, the group of isometries of $X$ is $\mathcal{G}=S O(4)$, and $X=\mathbb{S}^{3}$, the sphere. For all three models, the stationary subgroup of the point $(t, 0,0,0)$ is $\mathcal{K}=S O(3)$.

The surface $S(\chi)$ of a comoving sphere of radius $\chi$ is $S(\chi)=4 \pi f_{K}^{2}(\chi)$. The $\mathcal{G}$-invariant measure on $X$ in the above chart has the form

$$
d x=f_{K}^{2}(\chi) \sin \theta d \chi d \theta d \phi .
$$


The comoving distance $\chi$ runs from 0 to $\pi / \sqrt{K}$ when $K>0$, and from 0 to $\infty$ otherwise.

The scale factor, $a(t)$, is not observable. However, consider the electromagnetic wave of length $\lambda_{1}$ emitted by a distant galaxy at time $t_{1}<t_{0}$ that approaches the Earth now. It has another wave length, $\lambda_{0}$. The ratio $\lambda_{0} / \lambda_{1}$ is observable. It is denoted by

$$
\frac{\lambda_{0}}{\lambda_{1}}=1+z .
$$

The quantity $z$ is the celebrated redshift. Moreover,

$$
a\left(t_{1}\right)=\frac{1}{1+z} .
$$

In 1929, Hubble discovered that the redshift is positive for all but few close galaxies. It follows that $a\left(t_{1}\right)<1$. In other words, the Universe is expanding. The galaxies without redshift are those having big enough peculiar velocities to stand against the expansion in the standard chart.

Denote

$$
H_{0}=\dot{a}\left(t_{0}\right) \text {, }
$$

where a dot denote differentiation with respect to $t . H_{0}$ is called the Hubble constant. The number

$$
\rho_{c}=\frac{3 H_{0}^{2}}{8 \pi G}
$$

where $G$ is the Newton's gravitational constant, is called the critical density.

In general, $H(z)$ denote the Hubble constant as measured by an imaginary astronomer working at redshift $z$. The expansion of the Universe is governed by the Friedmann equation:

$$
H^{2}(z)=H_{0}^{2}\left[\Omega_{R}(1+z)^{4}+\Omega_{M}(1+z)^{3}+\Omega_{K}(1+z)^{2}+\Omega_{\Lambda}\right],
$$

where the parameters are as follows.

$\Omega_{M}$ is the density parameter for non-relativistic matter (which moves with speed much less than $c$ with respect to the fundamental observer). We have

$$
\Omega_{M}=\frac{\rho_{M, t_{0}}}{\rho_{c}},
$$

where $\rho_{M, t_{0}}$ is the density of the non-relativistic matter now, at $t=t_{0}$.

$\Omega_{\Lambda}$ is the vacuum energy parameter:

$$
\Omega_{\Lambda}=\frac{\Lambda c^{2}}{3 H_{0}^{2}}
$$

where $\Lambda$ is the cosmological constant.

$\Omega_{K}$ is the curvature parameter: 


$$
\Omega_{K}=-\frac{K c^{2}}{H_{0}^{2}} .
$$

Finally, $\Omega_{R}$ is the radiation density parameter. We have

$$
\Omega_{R}=\frac{\rho_{R, t_{0}}}{\rho_{c}}
$$

where $\rho_{R, t_{0}}$ is the density of the radiation now. When $z=0$, we obtain

$$
\Omega_{R}+\Omega_{M}+\Omega_{K}+\Omega_{\Lambda}=1
$$

The values for the above parameters, adapted from Efstathiou et al. (2014), are shown in Table 1, where 1 $\mathrm{Mpc}=3.09 \times 10^{26} \mathrm{~m}$, and the quoted errors show the $68 \%$ confidence level. The contribution from radiation is negligibly small, $\Omega_{R}=(4.9 \pm 0.5) \times 10^{-5}$.

Table 1

Cosmological parameters

\begin{tabular}{ll}
\hline Parameter & Value \\
\hline$H_{0}$ & $67.80 \pm 0.77 \mathrm{~km} / \mathrm{s} / \mathrm{Mpc}$ \\
$\Omega_{M}$ & $0.315_{-0.018}^{+0.016}$ \\
$\Omega_{K}$ & $-0.0010_{-0.0065}^{+0.0062}$ \\
$\Omega_{\Lambda}$ & $0.685_{-0.016}^{+0.018}$ \\
\hline
\end{tabular}

The comoving distance $\chi$ from the fundamental observer at $z=0$ to an object with redshift $z$ is

$$
\chi(z)=\frac{c}{H_{0}} \int_{0}^{z} \frac{d u}{\sqrt{\Omega_{R}(1+u)^{4}+\Omega_{M}(1+u)^{3}+\Omega_{K}(1+u)^{2}+\Omega_{\Lambda}}} .
$$

The look-back time $t_{L}$ to the above object is the difference between $t_{0}$ and the cosmic time at the moment when photons were emitted by the object. It is calculated as

$$
t_{L}=\frac{1}{H_{0}} \int_{0}^{z} \frac{d u}{(1+u) \sqrt{\Omega_{R}(1+u)^{4}+\Omega_{M}(1+u)^{3}+\Omega_{K}(1+u)^{2}+\Omega_{\Lambda}}} .
$$

In Friedmann equation, $a=0$ is reached after a finite time. Near $a=0$, the Hubble parameter $H(z)$ explodes. This point is therefore called Big Bang.

\section{Scalar fields}

\subsection{Deterministic Fields}

Let $f: M \rightarrow \mathbb{R}$ be a deterministic scalar cosmological field. On each time slice $X$, it is natural to decompose the restriction of $f$ to $X$ in eigenfunctions of $\mathcal{G}$-invariant differential operators.

Let $d(x, y)$ be a distance between the points $x$ and $y \in X$. In all three models, $X$ is a two-point homogeneous space. That is: for any two-point pairs $x, y \in X, x^{\prime}, y^{\prime} \in X$ satisfying 
$d(x, y)=d\left(x^{\prime}, y^{\prime}\right)$, there exists an isometry $g \in \mathcal{G}$ such that $g x=x^{\prime}, g y=y^{\prime}$. By (Helgason, 1984, Chapter 2, Proposition 4.11), all $\mathcal{G}$-invariant differential operators are polynomials in the Laplace-Beltrami operator $\Delta_{K}$ of the Riemannian manifold $X$. In what follows we call $\Delta_{K}$ just the Laplacian. We have

$$
\Delta_{K}=\frac{1}{f_{K}^{2}(\chi)} \frac{\partial}{\partial \chi}\left(f_{K}^{2}(\chi) \frac{\partial}{\partial \chi}\right)+\frac{1}{f_{K}^{2}(\chi)} \Delta,
$$

where

$$
\Delta=\frac{1}{\sin \theta} \frac{\partial}{\partial \theta}\left(\sin \theta \frac{\partial}{\partial \theta}\right)+\frac{1}{\sin ^{2} \theta} \frac{\partial^{2}}{\partial \varphi^{2}}
$$

is the Laplacian on the sphere $\mathbb{S}^{2}$.

The eigenvalue problem for the Laplacian $\Delta_{K}$ is traditionally written as the Helmholtz equation

$$
\Delta_{K} Q_{\mathbf{k}}(x)=-\left(k^{2}-K\right) Q_{\mathbf{k}}(x)
$$

where $\mathbf{k}$ is called the wave vector and labels the modes, $Q_{\mathbf{k}}(x)$, and where $k$ is called the wave number. The wave numbers take the values in $[0, \infty)$ for $K \leq 0$ and in the set $\left\{(\omega+1) \sqrt{K}: \omega \in \mathbb{Z}_{+}\right\}$otherwise, where $\mathbb{Z}_{+}$is the set of all nonnegative integers. Different authors use different normalisation of modes.

The Helmholtz equation may be solved by the method of separation of variables. We use the normalisation by Peter and Uzan (2009):

$$
Q_{k \ell m}(\chi, \theta, \varphi)=(2 \pi)^{3 / 2} R_{k \ell}(\chi) Y_{\ell m}(\theta, \varphi)
$$

where $Y_{\ell m}(\theta, \varphi)$ are spherical harmonics, the eigenfunctions of $\Delta$ :

$$
\Delta Y_{\ell m}=-\ell(\ell+1) Y_{\ell m}, \quad \ell \geq 0, \quad-\ell \leq m \leq \ell
$$

Spherical harmonics are related to associated Legendre polynomials $P_{\ell}^{m}$ through

$$
Y_{\ell m}(\theta, \varphi)=\sqrt{\frac{(2 \ell+1)(\ell-m) !}{4 \pi(\ell+m) !}} P_{\ell}^{m}(\cos \theta) e^{i m \varphi} .
$$

The radial part of the Helmholtz equation becomes

$$
\frac{1}{f_{K}^{2}(\chi)} \frac{d}{d \chi}\left(f_{K}^{2}(\chi) \frac{d R_{k \ell}(\chi)}{d \chi}\right)+\left[k^{2}-K-\frac{\ell(\ell+1)}{f_{K}^{2}(\chi)}\right] R_{k \ell}(\chi)=0
$$

When $K<0$, put $\omega=k / \sqrt{-K}$ and

$$
N_{k \ell}=\prod_{n=0}^{\ell}\left(\omega^{2}+n^{2}\right)
$$


The solution to (2) has the form

$$
R_{k \ell}(\chi)=\sqrt{\frac{\pi N_{k \ell}}{2 \omega^{2} \sinh (\sqrt{-K} \chi)}} P_{-1 / 2+i \omega}^{-1 / 2-\ell}(\cosh (\sqrt{-K} \chi)),
$$

where $P_{-1 / 2+i \omega}^{-1 / 2-\ell}$ is the associated Legendre function of the first kind.

When $K=0$, the solution to (2) has the form

$$
R_{k \ell}(\chi)=\sqrt{\frac{2}{\pi}} j_{\ell}(k \chi)
$$

where $j_{\ell}$ is the spherical Bessel function. The dimensionless distance in this case is $r=k \chi$.

Finally, when $K>0$, put $\omega=k / \sqrt{K}-1$ and

$$
M_{\omega \ell}=\prod_{n=0}^{\ell}\left((\omega+1)^{2}-n^{2}\right)
$$

Note that $M_{\omega \ell}=0$ for $\ell>\omega$. The solution to (2) has the form

$$
R_{\omega \ell}(\chi)=\sqrt{\frac{\pi M_{\omega \ell}}{2(\omega+1)^{2} \sin (\sqrt{K} \chi)}} P_{1 / 2+\omega}^{-1 / 2-\ell}(\cos (\sqrt{K} \chi)),
$$

see (Abbott and Schaefer, 1986, Equation (A21)).

For $K \leq 0$, the expansion of a function $f \in L^{2}(X, d x)$ into modes has the form

$$
f(\chi, \theta, \varphi)=(2 \pi)^{-3 / 2} \sum_{\ell=0}^{\infty} \sum_{m=-\ell}^{\ell} \int_{0}^{\infty} k^{2} f_{\ell m}(k) Q_{k \ell m}(\chi, \theta, \varphi) d k .
$$

For $K>0$, the above expansion reads

$$
f(\chi, \theta, \varphi)=\left(\frac{K}{2 \pi}\right)^{3 / 2} \sum_{\omega=0}^{\infty} \sum_{\ell=0}^{\omega} \sum_{m=-\ell}^{\ell}(\omega+1)^{2} f_{\ell m}(k) Q_{k \ell m}(\chi, \theta, \varphi),
$$

where the Fourier coefficients, $f_{\ell m}(k)$, are given by

$$
f_{\ell m}(k)=(2 \pi)^{-3 / 2} \int_{X} f(\chi, \theta, \varphi) \overline{Q_{k \ell m}(\chi, \theta, \varphi)} f_{K}^{2}(\chi) \sin \theta d \chi d \theta d \varphi .
$$

Assume that $K<0$ and $f$ depends only on $\chi$. Then we have

$$
Q_{k 00}(\chi)=\pi^{3 / 2} \frac{1}{\sqrt{\sin (\sqrt{-K} \chi)}} P_{-1 / 2+i \omega}^{-1 / 2}(\cosh (\sqrt{-K} \chi))
$$


Using the formula

$$
P_{-1 / 2+i \omega}^{-1 / 2}(\cosh r)=\sqrt{\frac{2}{\pi \sinh r}} \frac{\sin (\omega r)}{\omega}
$$

we obtain

$$
Q_{k 00}(\chi)=\pi \sqrt{2} \frac{\sqrt{-K} \sin (k \chi)}{k \sinh (\sqrt{-K} \chi)}
$$

and (5) becomes

$$
f(\chi)=\frac{1}{2 \sqrt{\pi}} \int_{0}^{\infty} f_{00}(k) \frac{\sqrt{-K} \sin (k \chi)}{k \sinh (\sqrt{-K} \chi)} k^{2} d k,
$$

where

$$
f_{00}(k)=\frac{1}{2 \sqrt{\pi}} \int_{0}^{\infty} f(\chi) \frac{\sqrt{-K} \sin (k \chi)}{k \sinh (\sqrt{-K} \chi)} S(\chi) d \chi .
$$

Similar calculations show that in the case of $K=0$ we have

$$
f(\chi)=\frac{1}{\pi \sqrt{2}} \int_{0}^{\infty} f_{00}(k) \frac{\sin (k \chi)}{k \chi} k^{2} d k,
$$

where

$$
f_{00}(k)=\frac{1}{\pi \sqrt{2}} \int_{0}^{\infty} f(\chi) \frac{\sin (k \chi)}{k \chi} S(\chi) d \chi,
$$

while in the case of $K>0$ we have

$$
f(\chi)=\frac{K^{3 / 2}}{2 \sqrt{\pi}} \sum_{\omega=0}^{\infty} f_{00}(\omega) \frac{\sin ((\omega+1) \sqrt{K} \chi)}{(\omega+1) \sin (\sqrt{K} \chi)}(\omega+1)^{2},
$$

where

$$
f_{00}(\omega)=\frac{1}{2 \sqrt{\pi}} \int_{0}^{\pi / \sqrt{K}} f(\chi) \frac{\sin ((\omega+1) \sqrt{K} \chi)}{(\omega+1) \sin (\sqrt{K} \chi)} S(\chi) d \chi .
$$

The above expansions, with various normalisations, were considered by Fock (1935), Schrodinger (1939, 1957), Lifshiz and Khalatnikov (1963), Harrison (1967), Abbott and Schaefer (1986), among others.

\subsection{Stochastic Fields}

Let $f: M \times \Omega \rightarrow \mathbb{C}$ be a stochastic scalar cosmological field defined on a probability space $(\Omega, \mathfrak{F}, \mathrm{P})$. We assume that $f$ has a finite variance, $\mathrm{E}\left[|f(t, x)|^{2}\right]<\infty$ and that $f$ is mean-square continuous, i.e., the map $M \rightarrow L^{2}(\Omega),(t, x) \mapsto f(t, x)$ is continuous, where $L^{2}(\Omega)$ is the Hilbert space of all random variables with finite variance. 
Consider the restriction $f(x)$ of the field $f(t, x)$ to the time slice $X$. By the cosmological principle, the field $f(x)$ must be $\mathcal{G}$-invariant, i.e., for any positive integer $n$, for any distinct points $x_{1}, \ldots$, $x_{n} \in X$, and for any $g \in \mathcal{G}$, random vectors $\left(f\left(x_{1}\right), \ldots, f\left(x_{n}\right)\right)^{\top}$ and $\left(f\left(g x_{1}\right), \ldots, f\left(g x_{n}\right)\right)^{\top}$ must have the same distribution.

In particular, the expected value $\mathrm{E}[f(x)]$ is a constant, say $a \in \mathbb{C}$, and the autocorrelation function

$$
R(x, y)=\mathrm{E}[(f(x)-a) \overline{(f(y)-a)}]
$$

is $\mathcal{G}$-invariant, i.e., $R(g x, g y)=R(x, y)$. Such fields are called wide-sense homogeneous. Note that $R(x, y)$ is a positive-definite function.

The spectral theory of wide-sense homogeneous random fields on topological groups and homogeneous spaces has been developed by Yaglom (1961). He proved spectral expansions of wide-sense homogeneous random fields on separable topological groups of type I and their homogeneous spaces. A topological group of type I is such a group $\mathcal{G}$ that every unitary representation of $\mathcal{G}$ generates an operator algebra of type I (see, for example, Naimark (1972)). All three groups $S O_{0}(1,3), I S O(3)$, and $S O(4)$, are of type I.

Moreover, the above groups share the following property. Let $U$ be an irreducible unitary representation of the group $\mathcal{G}$. Consider the restriction of $U$ to the compact subgroup $\mathcal{K}=S O(3)$. The above restriction is equivalent to a direct sum of irreducible unitary representations of $\mathcal{K}$. The multiplicity of the trivial representation of the group $\mathcal{K}$ in the above sum is equal either to 0 or to 1 .

Let $\hat{\mathcal{G}}_{\mathcal{K}}$ be the set of all equivalence classes of irreducible unitary representations of the group $\mathcal{G}$ for which the above multiplicity is equal to 1 . Let $U^{\omega} \in \hat{\mathcal{G}}_{\mathcal{K}}$, and let $H_{00}$ be the one-dimensional complex Hilbert space in which the trivial representation of the group $\mathcal{K}$ is realised. Let $\mathbf{e}$ be a vector of unit length in $H_{00}$. It is easy to see that the function

$$
\Phi_{\omega}(g)=\left(U^{\omega} \mathbf{e}, \mathbf{e}\right)_{H_{00}}
$$

does not depend on the choice of the vector $\mathbf{e}$. It is called the zonal spherical function of the group $\mathcal{G}$.

Let $\pi: \mathcal{G} \rightarrow X$ be the natural projection, let $x, y \in X$, and let $g_{1} \in \pi^{-1}(x), g_{2} \in \pi^{-1}(y)$. By (Yaglom, 1961, Theorem 6'), formula

$$
R(x, y)=\int_{\hat{\mathfrak{G}}_{\mathcal{K}}} \Phi_{\omega}\left(g_{2}^{-1} g_{1}\right) d v(\omega)
$$

determines a one-to-one correspondence between the autocorrelation functions $R(x, y)$ of wide-sense homogeneous random fields on $X$ and finite measures $v$ on $\hat{\mathcal{G}}_{\mathcal{K}}$. When $X$ is a two-point homogeneous space, the zonal spherical function $\Phi_{\omega}\left(g_{2}^{-1} g_{1}\right)$ depends only on $r$, the distance between $x$ and $y$.

The set $\hat{\mathcal{G}}_{\mathcal{K}}$ and the zonal spherical function for the case of $X=\mathbb{H}^{3}$ were determined by Gel'fand and Nalmark (1946). There exist different parametrisations of the above set. We will use the parametrisation by Kostant (1975). The set $\hat{\mathcal{G}}_{\mathcal{K}}$ includes the principal series parameterised by $\omega \in[0,+\infty)$, the supplementary series parameterised by $\omega \in(0, i)$, and trivial representation with $\omega=i$. We have

$$
\Phi_{\omega}(r)=\frac{\sin (\omega r)}{\omega \sinh r}
$$

and 


$$
R(x, y)=\int_{i}^{\infty} \frac{\sin (\omega r)}{\omega \sinh r} d v(\omega)
$$

where the integral is taken over the set $(0, i] \cup[0, \infty)$. Equation (9) was obtained by Krein (1949) for the case of a finite-dimensional hyperbolic space $\mathbb{H}^{n}$. To compare (9) with (6a), we choose $r$ in such a way that $\omega r=k \chi$, or $r=\sqrt{-K} \chi$. We obtain

$$
R(\chi)=\frac{1}{\sqrt{-K}} \int_{i \sqrt{-K}}^{\infty} \frac{\sqrt{-K} \sin (k \chi)}{k \sinh (\sqrt{-K} \chi)} d v(k) .
$$

Compare the last display with (6a). Apart from normalisation, we note the following difference. The expansion of a square-integrable function includes only the representations of the principal series of the group $\mathcal{G}$ (in physical terms, sub-curvature modes, when the wavelength is less than the radius of curvature). On the other hand, the expansion of a positive-definite function includes the representations of both the principal and the supplementary series (sub- and super-curvature modes, when the wavelength is greater than the radius of curvature). This difference was noted by Lyth and Woszczyna (1995). Lyth (1995) describes this situation as follows.

Mathematicians have known for almost half a century that all modes must be included to generate the most general homogeneous Gaussian random field, despite the fact that any square integrable function can be generated using only the sub-curvature modes. The former mathematical object, not the latter, is the relevant one for physical applications.

The set $\hat{\mathcal{G}}_{\mathcal{K}}$ and the zonal spherical functions for the case of $X=\mathbb{H}^{3}$ were determined by Krein (1949). We have $\hat{\mathcal{G}}_{\mathcal{K}}=[0, \infty)$,

$$
\Phi_{\omega}(r)=\frac{\sin (\omega r)}{\omega r}
$$

To compare this with (7a), choose $\omega=k$ and $r=\chi$. We obtain

$$
R(\chi)=\int_{0}^{\infty} \frac{\sin (k \chi)}{k \chi} d v(k)
$$

Cartan (1929) determined the set $\hat{\mathcal{G}}_{\mathcal{K}}$ and the zonal spherical functions for the case of $X=\mathbb{S}^{3}$. In this case $\hat{\mathcal{G}}_{\mathcal{K}}=\mathbb{Z}_{+}$,

$$
\Phi_{\omega}(r)=\frac{\sin ((\omega+1) r)}{(\omega+1) \sin r}
$$

To compare this with (8a), put $r=\sqrt{K} \chi$ and recall that $\omega=k / \sqrt{K}-1$. We obtain

$$
R(\chi)=\sum_{\omega=0}^{\infty} v(\omega) \frac{\sin ((\omega+1) \sqrt{K} \chi)}{(\omega+1) \sin (\sqrt{K} \chi)}
$$


where $v(\omega) \geq 0$ and $\sum_{\omega=0}^{\infty} v(\omega)<\infty$.

Equations (6), (7), and (8) have the following group-theoretical interpretation. Consider the set of double cosets $\mathcal{K} \backslash \mathcal{G} / \mathcal{K}$. When $K \leq 0$, this set is the interval $[0, \infty)$, otherwise this set is $[0, \pi \sqrt{K}]$. The measure $S(\chi) d \chi$ is a unique up to a constant multiplier $K$-bi-invariant measure on $\mathcal{K} / \mathcal{G} / \mathcal{K}$. For any choice of the above multiplier, say $C$, there exists a unique measure on $\hat{\mathcal{G}}_{\mathcal{K}}$ called the Plancherel measure. The Plancherel measure is characterised by the following property: equations (6)-(8) determine an isometric isomorphism between the space of $\mathcal{K}$-bi-invariant square-integrable functions on $\mathcal{G}$ with measure $C S(\chi) d \chi$ and the space of square-integrable functions on $\hat{\mathcal{G}}_{\mathcal{K}}$ with Plancherel measure. When $K \leq 0$, the measure $k^{2} d k$ is proportional to the Plancherel measure on $\hat{\mathcal{G}}_{\mathcal{K}}$. Note that the Plancherel measure is equal to zero for the supplementary series $\omega \in(0, i)$ and the trivial representation $\omega=i$. When $K>0$, the Plancherel measure of a point $\omega \in \hat{\mathcal{G}}_{\mathcal{K}}$ is proportional to $(\omega+1)^{2}$. Equations (6b), (7b), and (8b) define the spherical Fourier transform, while equations (6a), (7a), and (8a) define the inverse transform.

To find the spectral expansion of the random field $f$, we have to find a measurable space $(V, \mathfrak{B}, \mu)$ and a function $h: X \times V \rightarrow \mathbb{C}$ such that

$$
R(x, y)=\int_{V} h(x, v) \overline{h(y, v)} d \mu(v) .
$$

In this case there exists a complex-valued scattered random measure $Z$ on $V$ with $\mu$ as its control measure, i.e., for any $A, B \in \mathfrak{B}$ we have

$$
E[Z(A) \overline{Z(B)}]=\mu(A \cap B)
$$

such that

$$
f(x)=\int_{V} h(x, v) d Z(v) .
$$

Note that if $h(x, v)$ satisfies (13), then $h(x, v) e^{i \phi(v)}$ also satisfies the above equation.

For any $\omega \in \hat{\mathcal{G}}_{\mathcal{K}}$, consider the restriction of the representation $U^{\omega}$ to $\mathcal{K}$. In the cases of the open model with $\omega \neq i$ and the flat model with $\omega \neq 0$, the above restriction is equivalent to the direct sum of the irreducible unitary representations $D^{\ell}$ of the group $\mathcal{K}$ for all $\ell \in \mathbb{Z}_{+}$acting in Hilbert spaces $H_{\ell}$. In the case of the closed model, the above restriction is equivalent to the direct sum of the representations $D^{\ell}$, $0 \leq \ell \leq \omega$. The restriction of any representation $D^{\ell}$ to the subgroup $S O(2) \subset \mathcal{K}$ is equivalent to the direct sum of the representations $\phi \mapsto e^{i m \phi}$ of the group $S O(2),-\ell \leq m \leq \ell$. We represented the space $H_{\omega}$ of the representation $U^{\omega}$ as the direct sum of one-dimensional subspaces $H_{\ell m}$. Choose a vector $\mathbf{e}_{\ell m} \in H_{\ell m}$ of unit length in such a way, that the entries of matrices

$$
D_{m n}^{\ell}=\left(D^{\ell} \mathbf{e}_{\ell m}, \mathbf{e}_{\ell n}\right)_{H_{\ell}}
$$

are Wigner $D$-functions: 


$$
\begin{aligned}
D_{m n}^{\ell}(\phi, \theta, \psi)= & e^{-i(m \phi+n \psi)}(-1)^{m} \sqrt{\frac{(\ell+m) !(\ell-m) !}{(\ell+n) !(\ell-n) !}} \sin ^{2 \ell}(\theta / 2) \\
& \times \sum_{s=\max \{0, m+n\}}^{\min \{\ell+m, \ell+n\}}\left(\begin{array}{c}
\ell+n \\
s
\end{array}\right)\left(\begin{array}{c}
\ell-n \\
s-m-n
\end{array}\right)(-1)^{\ell-s+n} \cot ^{2 s-m-n}(\theta / 2) .
\end{aligned}
$$

Let $g=k_{1} r k_{2}$ be the standard polar decomposition of the elements of the group $G$. Choose $g_{i}=k_{i} r_{i} \in \pi^{-1}\left(x_{i}\right), i=1,2$. Then we have

$$
R(x, y)=\int_{\hat{G}_{K}} \Phi_{\omega}\left(r_{2}^{-1} k_{2}^{-1} k_{1} r_{1}\right) d v(\omega) .
$$

By matrix multiplication

$$
R(x, y)=\sum_{\ell} \sum_{m, n=-\ell}^{\ell} D_{m n}^{\ell}\left(k_{2}^{-1} k_{1}\right) \int_{\hat{G}_{K}} U_{\ell, m ; 0,0}^{\omega}\left(r_{1}\right) \overline{U_{\ell, n ; 0,0}^{\omega}\left(r_{2}\right)} d v(\omega) .
$$

The matrix entry $U_{\ell, m ; 0,0}^{\omega}\left(r_{1}\right)$ is called the associated spherical function. For all three models, they are different from 0 only when $m=0$. Therefore,

$$
R(x, y)=\sum_{\ell} D_{00}^{\ell}\left(k_{2}^{-1} k_{1}\right) \int_{\hat{G}_{K}} U_{\ell, 0 ; 0,0}^{\omega}\left(r_{1}\right) \overline{U_{\ell, 0 ; 0,0}^{\omega}\left(r_{2}\right)} d v(\omega) .
$$

Again by matrix multiplication

$$
R(x, y)=\sum_{\ell} \sum_{m=-\ell}^{\ell} D_{m 0}^{\ell}\left(k_{1}\right) \overline{D_{m 0}^{\ell}\left(k_{2}\right)} \int_{\hat{G}_{K}} U_{\ell, 0 ; 0,0}^{\omega}\left(r_{1}\right) \overline{U_{\ell, 0 ; 0,0}^{\omega}\left(r_{2}\right)} d v(\omega) .
$$

Wigner $D$-functions are related to spherical harmonics by

$$
Y_{\ell m}(\theta, \phi)=\sqrt{\frac{2 \ell+1}{4 \pi}} D_{-m 0}^{(\ell)}(\phi, \theta, 0) .
$$

Therefore we have

$$
\begin{aligned}
R(x, y)= & 4 \pi \sum_{\ell} \frac{1}{2 \ell+1} \sum_{m=-\ell}^{\ell} Y_{\ell m}\left(\theta_{1}, \phi_{1}\right) \overline{Y_{\ell m}\left(\theta_{2}, \phi_{2}\right)} \\
& \times \int_{\hat{G}_{K}} U_{\ell, 0 ; 0,0}^{\omega}\left(r_{1}\right) \overline{U_{\ell, 0 ; 0,0}^{\omega}\left(r_{2}\right)} d v(\omega),
\end{aligned}
$$

where

$$
r_{i}= \begin{cases}\sqrt{|K|} \chi_{i}, & K \neq 0 \\ k \chi_{i}, & K=0\end{cases}
$$

and $\left(\chi_{1}, \theta_{1}, \varphi_{1}\right)\left(\right.$ resp. $\left.\left(\chi_{2}, \theta_{2}, \varphi_{2}\right)\right)$ are coordinates of the point $x$ (resp. $y$ ) in the standard chart.

In the case of the open model we have

$$
V=\left\{((0, i] \cup[0, \infty), \ell, m): \ell \in \mathbb{Z}_{+},-\ell \leq m \leq \ell\right\},
$$

i.e., the union of countably many copies of the set $(0, i] \cup[0, \infty)$ indexed by pairs $(\ell, m), \mathfrak{B}$ is the $\sigma$ 
-field of Borel sets in $V$, and the restriction of the measure $\mu$ to any copy is equal to $v$.

The associated spherical functions for this case were calculated by Vilenkin (1958):

$$
U_{\ell, 0 ; 0,0}^{\omega}(r)=\frac{(-1)^{\ell} \sqrt{(2 \ell+1) \pi} \Gamma(i \omega)}{\sqrt{2} \Gamma(i \omega-\ell)} \sinh ^{-1 / 2} r P_{-1 / 2+i \omega}^{-1 / 2-\ell}(\cosh r) .
$$

Substitute this formula to (13). We obtain

$$
\begin{aligned}
R(x, y)= & 2 \pi^{2} \sum_{\ell=0}^{\infty} \sum_{m=-\ell}^{\ell} Y_{\ell m}\left(\theta_{1}, \phi_{1}\right) \overline{Y_{\ell m}\left(\theta_{2}, \phi_{2}\right)} \\
& \times \int_{i}^{\infty} \frac{|\Gamma(i \omega)|^{2} P_{-1 / 2+i \omega}^{-1 / 2-\ell}\left(\cosh \left(\sqrt{-K} \chi_{1}\right)\right) \overline{P_{-1 / 2+i \omega}^{-1 / 2-\ell}\left(\cosh \left(\sqrt{-K} \chi_{1}\right)\right)}}{|\Gamma(i \omega-\ell)|^{2} \sqrt{\sinh \left(\sqrt{-K} \chi_{1}\right) \sinh \left(\sqrt{-K} \chi_{2}\right)}} d v(\omega) .
\end{aligned}
$$

Using the formula $\Gamma(z)=(z-1) \Gamma(z-1)$ and (3), we have

$$
f(\chi, \theta, \phi)=2 \sqrt{\pi} \sum_{\ell=0}^{\infty} \sum_{m=-\ell}^{\ell} Y_{\ell m}(\theta, \phi) \int_{i \sqrt{-K}}^{\infty} R_{k \ell}(\chi) d Z_{\ell m}(k) .
$$

The case of the flat model is similar, but this time

$$
V=\left\{([0, \infty), \ell, m): \ell \in \mathbb{Z}_{+},-\ell \leq m \leq \ell\right\} .
$$

The associated spherical functions for this case were known in implicit form since Erdelyi et al. (1953). The group-theoretical interpretation has been proposed by Vilenkin (1957). We have

$$
U_{\ell, 0 ; 0,0}^{\omega}(r)=i^{\ell} \sqrt{2 \ell+1} j_{\ell}(\omega r)
$$

and the spectral expansion has the form

$$
f(\chi, \theta, \phi)=\pi \sqrt{2} \sum_{\ell=0}^{\infty} \sum_{m=-\ell}^{\ell} Y_{\ell m}(\theta, \phi) \int_{0}^{\infty} R_{k \ell}(\chi) d Z_{\ell m}(k) .
$$

Finally, in the case of the closed model

$$
V=\left\{(\omega, \ell, m): \omega \in \mathbb{Z}_{+}, 0 \leq \ell \leq \omega,-\ell \leq m \leq \ell\right\},
$$

$\boldsymbol{B}$ is the $\sigma$-field of all subsets of $V$, and $\mu(\omega, \ell, m)=v(\omega)$. The associated spherical functions are known since Erdelyi et al. (1953) and have the form

$$
U_{\ell, 0 ; 0,0}^{\omega}(r)=2^{\ell} \ell ! \sqrt{\frac{(\omega-\ell) !(2 \ell+1)}{(\omega+\ell+1) !(\omega+1)}} \sin ^{\ell} r C_{\omega-\ell}^{\ell+1}(\cos r),
$$

where $C_{\omega-\ell}^{\ell+1}(\cos r)$ are Gegenbauer polynomials. Using the formula

$$
C_{q}^{p}(\cos r)=\frac{\sqrt{\pi}(p+2 q-1) ! \sin ^{1 / 2-q} r}{2^{q-1 / 2}(q-1) ! p !} P_{p+q-1 / 2}^{1 / 2-q}(\cos r)
$$


the value $\omega=k / \sqrt{K}-1$, and (4), we obtain

$$
U_{\ell, 0 ; 0,0}^{\omega}(r)=\sqrt{2 \ell+1} R_{k \ell}(\chi)
$$

and

$$
f(\chi, \theta, \phi)=2 \sqrt{\pi} \sum_{\omega=0}^{\infty} \sum_{\ell=0}^{\omega} R_{k \ell}(\chi) \sum_{m=-\ell}^{\ell} Y_{\ell m}(\theta, \phi) Z_{k \ell m} .
$$

In what follows we suppose that the measure $v$ is absolutely continuous with respect to the Plancherel measure. In other words,

$$
R(\chi)= \begin{cases}\frac{1}{\sqrt{-K}} \int_{0}^{\infty} \frac{\sqrt{-K} \sin (k \chi)}{k \sinh (\sqrt{-K} \chi)} P(k) k^{2} d k, & K<0, \\ \int_{0}^{\infty} \frac{\sin (k \chi)}{k \chi} P(k) k^{2} d k, & K=0, \\ \frac{1}{K} \sum_{k / \sqrt{K}=1}^{\infty} \frac{\sqrt{-K} \sin (k \chi)}{k \sinh (\sqrt{-K} \chi)} P(k) k^{2}, & K>0,\end{cases}
$$

where $P(k)$ is the density of the measure $v$ with respect to the Plancherel measure. $P(k)$ is called the spectral density by mathematicians and the power spectrum by cosmologists. The power spectrum $P(k)$ is the spherical Fourier transforms of the autocorrelation function $R(\chi)$, while the latter is the inverse spherical Fourier transform of the power spectrum. We have the following theorem.

Theorem 1. The spectral expansion of the $\mathcal{G}$-invariant random field $f$ takes the following form. In the case of the open model

$$
f(\chi, \theta, \phi)=2 \sqrt{\pi} \sum_{\ell=0}^{\infty} \sum_{m=-\ell}^{\ell} Y_{\ell m}(\theta, \phi) \int_{0}^{\infty} R_{k \ell}(\chi) k \sqrt{P(k)} d W_{\ell m}(k) .
$$

In the case of the flat model

$$
f(\chi, \theta, \phi)=\pi \sqrt{2} \sum_{\ell=0}^{\infty} \sum_{m=-\ell}^{\ell} Y_{\ell m}(\theta, \phi) \int_{0}^{\infty} R_{k \ell}(\chi) k \sqrt{P(k)} d W_{\ell m}(k) .
$$

Finally, in the case of the closed model

$$
f(\chi, \theta, \phi)=2 \sqrt{\pi} \sum_{\omega=0}^{\infty} \sum_{\ell=0}^{\omega} R_{\omega \ell}(\chi) \sum_{m=-\ell}^{\ell} Y_{\ell m}(\theta, \phi) \omega \sqrt{P(\omega)} W_{\omega \ell m},
$$

where $W_{\ell m}$ is a sequence of uncorrelated identically distributed complex-valued scattered random measures on $[0, \infty)$ with Lebesgue measure as their common control measure, and where $W_{\omega t m}$ is a sequence of uncorrelated identically distributed random variables with zero mean and unit variance.

\section{Spin Fields}

In order to motivate the introducing of spin fields, consider the following example. Let $\rho(t, x)$, $x=(\chi, \theta, \varphi) \in X$ be the density of the non-relativistic matter. Currently, only the baryonic part of $\rho$ is 
observable. Define the fractional overdensity as

$$
\delta(t, x)=\frac{\rho(t, x)-\rho_{M, t}}{\rho_{M, t}} .
$$

The Newton's gravitational potential, $\phi$, is the solution to Poisson's equation

$$
\Delta_{K} \phi(t, x)=\frac{4 \pi G}{c^{2}} \rho(t, x) .
$$

Consider a source of electromagnetic waves at the comoving distance $\chi$ with angular coordinates $\theta$ and $\varphi$. The effective lensing potential of the source is defined as

$$
\psi(\chi, \theta, \varphi)=\frac{2}{f_{K}(\chi)} \int_{0}^{\chi} \frac{f_{K}(\chi-r)}{f_{K}(r)} \varphi\left(t_{0}-t_{L}, r, \theta, \varphi\right) d r .
$$

This quantity is not observable. However, the convergence $\kappa=(1 / 2)$ ð $\psi$ and the shear $\gamma=(1 / 2)$ $ð^{2} \psi$ are observable now, while the first gravitational flexion $F=(1 / 2) \partial{ }^{*} \succsim \psi$ and the second gravitational flexion $G=(1 / 2) \delta^{3} \psi$ may become observable in the near future.

The symbol $\partial$ denote the spin raising operator. There exist several equivalent definitions of this object. Consider the one connected to representation theory.

Let $s \in \mathbb{Z}$ be an integer number. The map $s \mapsto\left(\varphi \mapsto e^{i s \phi}\right), \varphi \in S O(2)$ determines a one-to-one correspondence between $\mathbb{Z}$ and the set of the irreducible unitary representations of the group $\mathcal{H}=S O(2)$. Consider the Cartesian product $S O(3) \times \mathbb{C}$ and introduce the following equivalence relation: the points $\left(g_{1}, z_{1}\right)$ and $\left(g_{2}, z_{2}\right)$ are equivalent if there exists an element $h=h_{\phi} \in \mathcal{H}$ such that $g_{2}=g_{1} h$ and $z_{2}=e^{-i s \phi} z_{1}$. Call the quotient space $E_{s}$. The projection map $\pi(g, z)=g \mathcal{H}$ maps $E_{s}$ onto the homogeneous space $S O(3) / S O(2)$, which is the two-dimensional sphere, $\mathbb{S}^{2}$. The inverse image of each point in $\mathbb{S}^{2}$ is a copy of $\mathbb{C}$. The triple $\left(E_{s}, \pi, \mathbb{S}^{2}\right)$ is a homogeneous line bundle over $\mathbb{S}^{2}$.

Let $f: \mathbb{S}^{2} \rightarrow E_{s}$ be a section of the above bundle, i.e., $\pi \circ f$ is the identity map on $\mathbb{S}^{2}$. The group $S O(3)$ acts on $E_{s}$ by $g\left(g_{0}, z\right)=\left(g g_{0}, z\right)$. This action identifies the fibers over any two points of the base space. Therefore, we can define the square-integrable sections by the condition

$$
\int_{0}^{2 \pi} \int_{0}^{\pi}|f(\theta, \phi)|^{2} \sin \theta d \theta d \phi<\infty
$$

The elements of this space are called the square-integrable functions of spin $S$.

The induced representation $U$ of the group $S O(3)$ acts in the space $L_{2}\left(E_{s}\right)$ of the square-integrable sections by

$$
U(g) f(n)=f\left(g^{-1} n\right), \quad g \in S O(3), \quad n \in \mathbb{S}^{2} .
$$

The Frobenius reciprocity states that the multiplicity of the irreducible representation $U_{\ell}$ in $U$ is equal to the multiplicity of the representation $s$ in $U_{\ell}$. The latter is equal to 1 if $|s| \leq \ell$ and to 0 otherwise. It follows that the induced representation $U$ is equivalent to the direct sum of irreducible components $U_{\ell}$, 
$\ell=|s|,|s|+1, \ldots$.

The basis in the space $L_{2}\left(E_{0}\right)$ consists of spherical harmonics (15) (of spin 0). The basis in $L_{2}\left(E_{s}\right)$ consists of spherical harmonics of spin $s$ :

$$
{ }_{s} Y_{\ell m}(\theta, \phi)=\sqrt{\frac{2 \ell+1}{4 \pi}} D_{-m,-s}^{(\ell)}(\phi, \theta, 0) .
$$

The last display and (11) imply the following formula:

$$
\begin{aligned}
{ }_{s} Y_{\ell m}(\theta, \phi)= & e^{i m \phi}(-1)^{m} \sqrt{\frac{(2 \ell+1)(\ell+m) !(\ell-m) !}{4 \pi(\ell+s) !(\ell-s) !}} \sin ^{2 \ell}(\theta / 2) \\
& \times \sum_{u=\max \{0, m-s\}}^{\min \{\ell+m, \ell-s\}}\left(\begin{array}{c}
\ell-s \\
u
\end{array}\right)\left(\begin{array}{c}
\ell+s \\
u-m+s
\end{array}\right)(-1)^{\ell-u-s} \cot ^{2 u-m+s}(\theta / 2) .
\end{aligned}
$$

Consider the operator $\circlearrowright$ (in fact, a family of operators) acting onto a spherical harmonic of spin $s$ by

$$
ð_{s} Y_{\ell m}=\sqrt{(\ell-s)(\ell+s+1)} Y_{s+1} .
$$

This action may be extended by linearity to the linear subset $\mathcal{D}_{ð}$ of the space $L_{2}\left(E_{s}\right)$ that consists of the functions ${ }_{s} f$, for which their Fourier coefficients

$$
{ }_{s} f_{\ell m}=\int_{S^{2} s} f(\theta, \varphi) \overline{Y_{\ell m}(\theta, \varphi)} \sin \theta d \theta d \varphi
$$

satisfy

$$
\left.\left.\sum_{\ell=|s|+1}^{\infty}(\ell-s)(\ell+s+1)\right|_{s} f_{\ell m}\right|^{2}<\infty .
$$

It is easy to check, that

$$
ð=s \cot \theta-\frac{\partial}{\partial \theta}-\frac{i}{\sin \theta} \frac{\partial}{\partial \varphi} .
$$

The conjugate operator, $\partial^{*}$, acts onto a spherical harmonic of spin $s$ by

$$
\partial^{*} Y_{\ell m}=-\sqrt{(\ell+s)(\ell-s+1)}{ }_{s-1} Y_{\ell m} .
$$

By obvious reasons, $\partial$ is called the spin raising operator, while $\partial^{*}$ is called the spin lowering operator. Moreover, the restrictions of these operators to the space $H_{\ell}$, where the irreducible representation $U_{\ell}$ acts, are intertwining operators between equivalent representations.

Assume that the effective lensing potential of the sources of radiation is a single realisation of a random field. By the cosmological principle, the above field must be $\mathcal{G}$-invariant. In particular, its spectral expansion is determined by Theorem 1. Consider the case of the convergence field in an open model. Assume that the power spectrum $P(k)$ satisfies the following condition: 


$$
\sum_{\ell=1}^{\infty}[\ell(\ell+1)(2 \ell+1)]^{2} \int_{0}^{\infty}\left|R_{k \ell}(\chi)\right|^{2} k^{2} P(k) d k<\infty .
$$

Apply operator $(1 / 2)^{*} ð^{*}$ ð to the expansion (14) term by term:

$$
\kappa(\chi, \theta, \varphi)=-\sqrt{\pi} \sum_{\ell=1}^{\infty} \sum_{m=-\ell}^{\ell} \ell(\ell+1) Y_{\ell m}(\theta, \varphi) \int_{0}^{\infty} R_{k \ell}(\chi) k \sqrt{P(k)} d W_{\ell m}(k)
$$

The series in the right hand side converges in mean square and determines the spectral expansion of a random field. Note that the above expansion cannot be written in the form of (14). Therefore, the random field $\kappa(\chi, \theta, \varphi)$ is not $S O_{0}(1,3)$-invariant. However, the restriction of the field $\kappa$ to the sphere of a fixed comoving radius $\chi_{0}$ centred at the fundamental observer is an isotropic random field on the sphere. In other words, the above restriction is an isotropic random section of the line bundle $\left(E_{0}, \pi, \mathbb{S}^{2}\right)$.

The heuristic arguments of the previous paragraph suggest the following. The restriction of the convergence field to the sphere of a fixed comoving radius $\chi_{0}$ centred at the fundamental observer is an isotropic random section of the line bundle $\left(E_{0}, \pi, \mathbb{S}^{2}\right)$, that of the first gravitational flexion field is an isotropic random section of the line bundle $\left(E_{1}, \pi, \mathbb{S}^{2}\right)$, that of the shear field is an isotropic random section of the line bundle $\left(E_{2}, \pi, \mathbb{S}^{2}\right)$. Finally, the restriction of the second gravitational flexion field to the sphere of a fixed comoving radius $\chi_{0}$ centred at the fundamental observer is an isotropic random section of the line bundle $\left(E_{3}, \pi, \mathbb{S}^{2}\right)$. This means that the expected value $\mathrm{E}\left[X\left(\chi_{0}, \mathbf{n}\right)\right], X \in\{\kappa, \gamma, F, G\}, \mathbf{n} \in S^{2}$, is constant, while the covariance function $\mathbf{E}\left[X\left(\chi_{0}, \mathbf{n}_{1}\right) \overline{X\left(\chi_{0}, \mathbf{n}_{2}\right)}\right]$ satisfies the following condition:

$$
\mathbf{E}\left[X\left(\chi_{0}, g \mathbf{n}_{1}\right) \overline{X\left(\chi_{0}, g \mathbf{n}_{2}\right)}\right]=\mathbf{E}\left[X\left(\chi_{0}, \mathbf{n}_{1}\right) \overline{X\left(\chi_{0}, \mathbf{n}_{2}\right)}\right], \quad g \in S O(3) .
$$

Spectral expansion of an isotropic random field in the trivial bundle $\left(E_{0}, \pi, \mathbb{S}^{2}\right)$ goes back to Obukhov (1947), that in the bundle $\left(E_{2}, \pi, \mathbb{S}^{2}\right)$ is known to cosmologists since Zaldarriaga and Seljak (1997). The rigourous mathematical theory of isotropic random fields in vector bundles was proposed by Geller and Marinucci (2010) and Malyarenko (2011). In particular, the spectral expansion of an isotropic random section $X(\theta, \phi)$ of the line bundle $\left(E_{s}, \pi, \mathbb{S}^{2}\right), s \in \mathbb{Z}$, has the form

$$
X(\theta, \varphi)=\sum_{\ell=s}^{\infty} \sum_{m=-\ell}^{\ell} a_{s, \ell m s} Y_{\ell m}(\theta, \varphi)
$$

where $\mathrm{E}\left[a_{s, \ell m}\right]=0$ unless $s=\ell=0$ and

$$
\begin{gathered}
\mathrm{E}\left[a_{s, \ell m} \overline{a_{s, \ell^{\prime} m^{\prime}}}\right]=\delta_{\ell \ell^{\prime}} \delta_{m m^{\prime}} C_{s \ell}, \\
\sum_{\ell=s}^{\infty}(2 \ell+1) C_{s \ell}<\infty .
\end{gathered}
$$

It follows that there exists a sequence of uncorrelated mean square continuous stochastic processes $a_{s, \ell m}(\chi)$ such that 


$$
X(\chi, \theta, \phi)=\sum_{\ell=s}^{\infty} \sum_{m=-\ell}^{\ell} a_{s, \ell m}(\chi)_{s} Y_{\ell m}(\theta, \phi) .
$$

Moreover, $\mathrm{E}\left[a_{s, \ell m}(\chi)\right]=0$ unless $s=\ell=m=0$ and

$$
\mathrm{E}\left[a_{s, \ell m}\left(\chi_{1}\right) \overline{a_{s, \ell^{\prime} m^{\prime}}\left(\chi_{2}\right)}\right]=\delta_{\ell \ell^{\prime}} \delta_{m m^{\prime}} C_{s \ell}\left(\chi_{1}, \chi_{2}\right)
$$

with

$$
\sum_{\ell=s}^{\infty}(2 \ell+1) C_{s \ell}(\chi, \chi)<\infty .
$$

As usual, the comoving distance $\chi$ runs from 0 to $\pi / \sqrt{K}$ when $K>0$, and from 0 to $\infty$ otherwise.

The correlation function of the random field (18) is

$$
\begin{aligned}
R\left(\chi_{1}, \theta_{1}, \phi_{1}, \chi_{2}, \theta_{2}, \phi_{2}\right) & =E\left[X\left(\chi_{1}, \theta_{1}, \phi_{1}\right) \overline{X\left(\chi_{2}, \theta_{2}, \phi_{2}\right)}\right] \\
& =\sum_{\ell=s}^{\infty} C_{s \ell}\left(\chi_{1}, \chi_{2}\right) \overline{\sum_{m=-\ell}^{\ell}{ }_{s} Y_{\ell m}\left(\theta_{1}, \phi_{1}\right) \overline{Y_{\ell m}\left(\theta_{2}, \phi_{2}\right)} .}
\end{aligned}
$$

To simplify this formula, use the addition theorem for spin weighted spherical harmonics from (Durrer, 2008, equation (A4.57)):

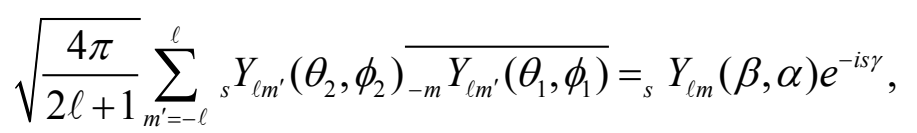

where $(\alpha, \beta, \gamma)$ are the Euler angles of the rotation which first rotates $\left(\theta_{2}, \varphi_{2}\right)$ into the north pole and then north pole into $\left(\theta_{1}, \varphi_{1}\right)$. In fact, $\beta$ is the angle between $\left(\theta_{1}, \varphi_{1}\right)$ and $\left(\theta_{2}, \varphi_{2}\right), \alpha$ is the angle between the great arc connecting the above two points and the meridian passing through $\left(\theta_{1}, \varphi_{1}\right)$, and $\gamma$ is the angle between the great arc connecting the above two points and the meridian passing through $\left(\theta_{2}, \varphi_{2}\right)$, see Fig. 1 in $\mathrm{Ng}$ and Liu (1999). Substitute the value $m=-s$, use formula

$$
{ }_{s} Y_{\ell m}(\beta, \alpha)=e_{s}^{-i m \alpha} Y_{\ell m}(\beta, 0),
$$

and interchange indices 1 and 2 . We obtain

$$
\sum_{m=-\ell}^{\ell}{ }_{s} Y_{\ell m}\left(\theta_{1}, \varphi_{1}\right)_{s} \overline{Y_{\ell m}\left(\theta_{2}, \varphi_{2}\right)}=\sqrt{\frac{2 \ell+1}{4 \pi}} Y_{\ell}(\beta, 0) e^{-i s(\alpha+\gamma)},
$$

and

$$
\boldsymbol{R}\left(\chi_{1}, \theta_{1}, \varphi_{1}, \chi_{2}, \theta_{2}, \varphi_{2}\right)=\frac{1}{2 \sqrt{\pi}} \sum_{\ell=s}^{\infty} C_{s \ell}\left(\chi_{1}, \chi_{2}\right) \sqrt{2 \ell+1} Y_{\ell-s}(\beta, 0) e^{-i s(\alpha+\gamma)}
$$

To calculate $C_{s \ell}\left(\chi_{1}, \chi_{2}\right)$, put $\theta_{1}=\varphi_{2}=0$ and $\theta_{2}=\beta$ in the last display. In other words, let 
$\left(\theta_{1}, \varphi_{1}\right)$ be the north pole. Then $\alpha=\gamma=0$ and

$$
\boldsymbol{R}\left(\chi_{1}, 0,0, \chi_{2}, \beta, 0\right)=\frac{1}{2 \sqrt{\pi}} \sum_{\ell=s}^{\infty} C_{s \ell}\left(\chi_{1}, \chi_{2}\right) \sqrt{2 \ell+1}_{s} Y_{\ell-s}(\beta, 0) .
$$

The spin weighted spherical harmonics ${ }_{s} Y_{\ell m}(\theta, \varphi), \ell \geq|s|,-\ell \leq m \leq \ell$ form an orthonormal basis in the space of square integrable functions on the sphere $\mathbb{S}^{2}$. It follows from (18) that the functions $\sqrt{2 \pi} Y_{\ell-s}(\theta, 0)$ form an orthonormal basis in the space $L^{2}([0, \pi], \sin \theta d \theta)$. Multiply both hand sides of the last display by $\overline{{ }_{s}(\beta, 0)}$ and integrate over $[0, \pi]$ with respect to the measure $\sin \beta d \beta$. We obtain

$$
C_{s \ell}\left(\chi_{1}, \chi_{2}\right)=\frac{4 \pi^{3 / 2}}{\sqrt{2 \ell+1}} \int_{0}^{\pi} R\left(\chi_{1}, 0,0, \chi_{2}, \beta, 0\right) \overline{Y_{\ell-s}(\beta, 0)} \sin \beta d \beta .
$$

Multiply both hand sides of equation (18) by $\overline{{ }_{s} Y_{\ell m}(\theta, \varphi)}$ and integrate over $\mathbb{S}^{2}$. We obtain

$$
a_{s, \ell m}(\chi)=\int_{S^{2}} X(\chi, \theta, \varphi) \overline{Y_{\ell m}(\theta, \varphi)} \sin \theta d \theta d \varphi .
$$

It follows from mean square continuity that

$$
\lim _{\chi \downarrow 0} \mathrm{E}\left[\left|a_{s, \ell m}(\chi)\right|^{2}\right]=0, \quad(s, \ell, m) \neq(0,0,0),
$$

therefore

$$
C_{s \ell}(0, \chi)=0, \quad \ell \neq 0 .
$$

We arrive at the following theorem.

Theorem 2. The spin cosmological fields have the form (15), where $s=0$ for the convergence, $s=1$ for the first gravitational flexion, $s=2$ for the shear, and $s=3$ for the second gravitational flexion. The sequence of stochastic processes $a_{s, \ell m}(\chi)$ satisfies (16) and (17). The correlation function of a spin cosmological field has the form (19), where $C_{s \ell}\left(\chi_{1}, \chi_{2}\right)$ is a sequence of nonnegative-definite kernels satisfying (17) and (21). The kernels $C_{s \ell}\left(\chi_{1}, \chi_{2}\right)$ may be calculated by (20).

Theorem 2 in the case of $s=0$ is due to Yadrenko (1983).

\section{References}

L. Abbott and R. Schaefer. A general, gauge-invariant analysis of the cosmic microwave anisotropy. Astroph. J., 308:546-562, 1986.

E. Cartan. Sur la de'termination d'un syste'me orthogonal complet dans un espace de Riemann symme'trique clos. Rend. Circ. Mat. Palermo, 53:217-252, 1929.

R. Durrer. The cosmic microwave background. Cambridge University Press, 2008.

G. Efstathiou. Planck 2013 results. XVI. Cosmological parameters. A\&A, 571:1-66, 2014.

A. Erdelyi, W. Magnus, F. Oberhettinger, and F. G. Tricomi. Higher transcendental functions, volume II. McGraw-Hill, New York, 1953.

V. Fock. Zur Theorie des Wasserstoffatoms. Zeitschrift für Physik, 98:145-154, March 1935. doi: 10.1007/BF01336904.

I. M. Gel'fand and M. A. Na1mark. Unitary representations of the Lorentz group. Acad. Sci. USSR. J. Phys., 10:93-94, 1946.

D. Geller and D. Marinucci. Spin wavelets on the sphere. J. Fourier Anal. Appl., 16:840-884, 2010.

E. Harrison. Normal modes of vibrations of the Universe. Rev. Mod. Phys., 39(4):862-882, 1967. 
S. Helgason. Groups and geometric analysis. Integral geometry, invariant differential operators, and spherical functions. Pure and applied mathematics. Academic Press, Orlando, 1984.

B. Kostant. On the existence and irreducibility of certain series of representations. In I. Gel'fand, editor, Lie groups and their representations, pages 231-329. Halsted Press, New York, 1975.

M. Kreın. Hermitian positive kernels on homogeneous spaces. Ukrain.Mat. Z urnal, 1(4):64-98, 1949.

E. Lifshitz and I. Khalatnikov. Investigations in relativistic cosmology. Advances in Phys., 12:185-249, 1963.

D.H. Lyth. The Grishchuk-Zeldovich effect in the open Universe. Annals N.Y. Acad. Sci., 759:701, 1995.

D.H. Lyth and A. Woszczyna. Large scale perturbations in the open Universe. Phys. Rev. D, 52:3338-3357, 1995.

A. Malyarenko. Invariant random fields in vector bundles and application to cosmology. Ann. Inst. H. Poincare' Probab. Stat., 47(4):1068-1095, 2011. doi: 10.1214/10-AIHP409.

M. A. Na1mark. Normed algebras. Wolters-Noordhoff Ser. Monogr. Text. Pure Appl. Math. Wolters- Noordhoff Publishing, Groningen, third edition, 1972.

K.-W. Ng and G.-C. Liu. Correlation functions of CMB anisotropy and polarization. Int. J. Mod. Phys. D, 8 (1):61-83, 1999.

A. M. Obukhov. Statistically homogeneous random fields on a sphere. Uspehi Mat. Nauk, 2(2):196-198, 1947. Russian.

P. Peter and J.-P. Uzan. Primordial cosmology. Oxford University Press, Oxford, 2009.

E. Schrodinger. The proper vibrations of the expanding Universe. Physica, 6:899-912, 1939.

E. Schrodinger. Expanding Universes. Cambridge University Press, Cambridge, 1957.

N. Ya. Vilenkin. The matrix elements of irreducible unitary representations of the group of real orthogonal matrices and group of Euclidean (n-1)-dimensional space motions. Dokl. Akad. Nauk SSSR (N.S.), 113: 16-19, 1957. Russian.

N. Ya. Vilenkin. The matrix elements of irreducible unitary representations of a group of Lobachevsky space motions and the generalized Fock-Mehler transformations. Dokl. Akad. Nauk SSSR (N.S.), 118:219-222, 1958. Russian.

H. Weyl. Zur allgemeinen Relativittstheorie. Physikalische Zeitschrift, 29: 230-232, 1923.

M. I. Yadrenko. Spectral theory of random fields. Translat. Ser. Math. Eng. Optimization Software, Publications Division, New York, 1983.

A. M. Yaglom. Second-order homogeneous random fields. In Proc. 4th Berkeley Sympos. Math. Statist. and Probab., volume II, pages 593-622, Berkeley, CA, 1961. Univ. California Press.

M. Zaldarriaga and U. Seljak. An all-sky analysis of polarisation in the microwave background. Phys. Rev. D, 55 (4):1830-1840, 1997. 